\title{
Recent results from the Hamiltonian approach to Yang-Mills theory in Coulomb gauge
}

\author{
H. Reinhardt; G . Burgio, D. Campagnari, D. Epple, M. Leder, M. Pak, M. Quandt \\ and W. Schleifenbaum \\ University of Tübingen \\ Institute of Theoretical Physics \\ Auf der Morgenstelle 14 \\ D-72076 Tübingen \\ Email: hugo.reinhardt@uni-tuebingen.de
}

\begin{abstract}
Within the Hamiltonian approach to Yang-Mills theory in Coulomb gauge the ghost and gluon propagators are determined from a variational solution of the Yang-Mills Schrödinger equation showing both gluon and heavy quark confinement. The continuum results are in good agreement with lattice data. The ghost form factor is identified as the dielectric function of the Yang-Mills vacuum and the Gribov-Zwanziger confinement scenario is shown to imply the dual Meissner effect. The topological susceptibility is calculated.
\end{abstract}

8th Conference Quark Confinement and the Hadron Spectrum

September 1-62008

Mainz, Germany

\footnotetext{
${ }^{*}$ Speaker.

${ }^{\dagger}$ supported by DFG-Re 856/6-2,3
} 

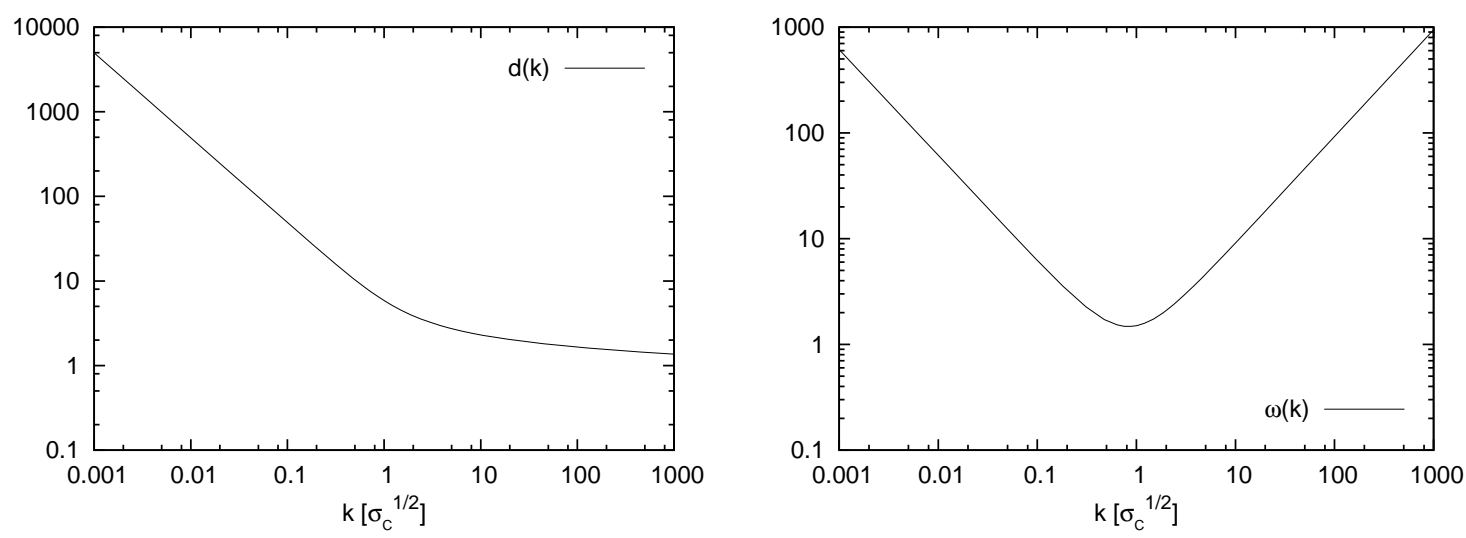

Figure 1: Ghost form factor $d(k)$ (left) and gluon energy $\omega(k)$ from the variational solutions presented in [1].

We solve the Schrödinger equation $H \psi=E \psi$ of Yang-Mills theory in Coulomb gauge by the variational principle $\langle\psi|H| \psi\rangle \rightarrow$ min with the following ansatz for the vacuum wave functional $\psi\left(A^{\perp}\right)[3]$.

$$
\psi\left(A^{\perp}\right)=\frac{1}{\sqrt{J\left(A^{\perp}\right)}} \exp \left(-\frac{1}{2} \int d^{3} x d^{3} y A_{i}^{\perp a}(x) \omega(x, y) A_{i}^{\perp a}(y)\right),
$$

where the kernel $\omega(x, y)$ is determined from the variational principle [1], [2], [3] and $J\left(A^{\perp}\right)=$ $\operatorname{Det}(-\hat{D} \partial)$ is the Faddeev-Popov determinant. In practice the so resulting equation for $\omega(x, y)$ is converted into a set of Dyson-Schwinger equations for the gluon propagator

$$
\left\langle A_{i}^{\perp a}(x) A_{j}^{\perp b}(y)\right\rangle=\delta^{a b} t_{i j}(x) \frac{1}{2} \omega^{-1}(x, y),
$$

with $t_{i j}(x)=\delta_{i j}-\frac{\partial_{i} \partial_{j}}{\partial^{2}}$ being the transverse projector, and the ghost propagator

$$
G(x, y)=\left\langle(-\hat{D} \cdot \partial)^{-1}\right\rangle=\left\langle x\left|d(-\Delta)(-\Delta)^{-1}\right| y\right\rangle .
$$

Here we have introduced the ghost form factor $d(-\Delta)$, which describes the deviation of the QCD ghost propagator from the QED case, where $d(-\Delta) \equiv 1$. The resulting Dyson-Schwinger equations need renormalisation, which is well under control [15]. Fig. 1 shows the solution of the DysonSchwinger equation for the gluon energy $\omega(k)$ and the ghost form factor $d(k)$, as shown in Ref. [1]. An analytic infrared and ultraviolet analysis of the Dyson-Schwinger equation shows the following asymptotic behaviour $[3,4]$

$$
\begin{array}{rlrl}
\operatorname{IR}(k \rightarrow 0): \omega(k) & \sim \frac{1}{k} & d(k) & \sim \frac{1}{k} \\
\mathrm{UV}(k \rightarrow \infty): \omega(k) & \sim k & d(k) & \sim k^{0} .
\end{array}
$$

At large momenta the gluon behaves like a photon, which is in agreement with asymptotic freedom, while at small momenta the gluon energy diverges, which implies the absence of gluon states in the physical spectrum. This is nothing but a manifestation of gluon confinement. The infrared divergence of the ghost form factor is a consequence of the horizon condition

$$
d^{-1}(k=0)=0
$$



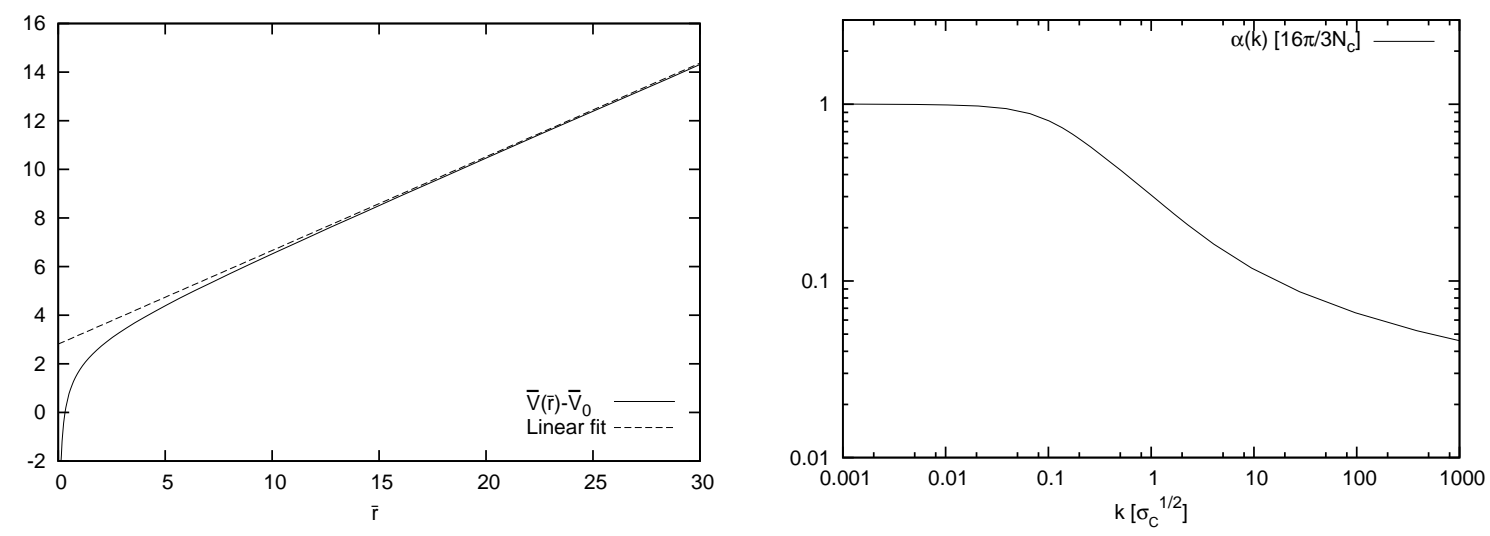

Figure 2: Left: Heavy quark potential given by eq. (6). Right: Running coupling constant.

which has been used as input in the renormalisation of the ghost Dyson-Schwinger equation. This is a necessary condition for the Gribov-Zwanziger confinement scenario. In fact, one can show that there is a sum rule relating the infrared exponents of the ghost and the gluon propagator and an infrared divergent gluon energy requires also an infrared divergent ghost form factor, i.e. the horizon condition (5), see Ref. [4]. A similar behaviour of the propagators is also obtained from functional renormalization group flow equations [5]. Fig. 2 shows the non-Abelian Coulomb potential

$$
V(|x-y|)=g^{2}\left\langle\left\langle x\left|(-\hat{D} \cdot \partial)^{-1}\left(-\partial^{2}\right)(-\hat{D} \cdot \partial)^{-1}\right| y\right\rangle\right\rangle \stackrel{|x-y| \rightarrow \infty}{\longrightarrow} \sigma_{C}|x-y|,
$$

which for large distances indeed increases linearly [1] as the infrared analysis reveals. The Coulomb string tension $\sigma_{C}$ sets the scale of our approach. Also shown in Fig. 2 is the running coupling constant which is infrared finite, for details see Ref. [4].

In $D=3+1$ dimensions, previous lattice calculations performed in Coulomb gauge in Ref. $[7,8]$ showed an anomalous UV behaviour of the gluon propagator - IR: $\omega(k) \sim k^{0}$, UV: $\omega(k) \sim$ $k^{3 / 2}$ - which is in strong conflict with the continuum result. However, one should mention that these lattice calculations assumed multiplicative renormalisability of the 4-dimensional gluon propagator, which give rise to scaling violations in the static propagator. Furthermore, these calculations did not fix the gauge completely, i.e. the residual time-dependent gauge invariance left after Coulomb gauge fixing was left unfixed.

Recently, we have done improved lattice calculations with a complete gauge fixing [10]. In these studies, the energy dependence of the 4-dimensional gluon propagator could be explicitly extracted and it was found that the static gluon propagator is multiplicatively renormalisable and shows a perfect scaling. Fig. 3 (left panel) shows the results for the gluon propagator of these calculations together with the continuum results. It is assumed here that the Coulomb string tension $\sigma_{C}$ is identical to the string tension $\sigma$ from the Wilson loop. There is a good agreement, particularly in the infrared and ultraviolet the lattice and continuum results match perfectly. It is also remarkable that the lattice result can be very well fitted by Gribov's original formula for the gluon energy

$$
\omega(k)=\sqrt{k^{2}+\frac{M^{4}}{k^{2}}}
$$



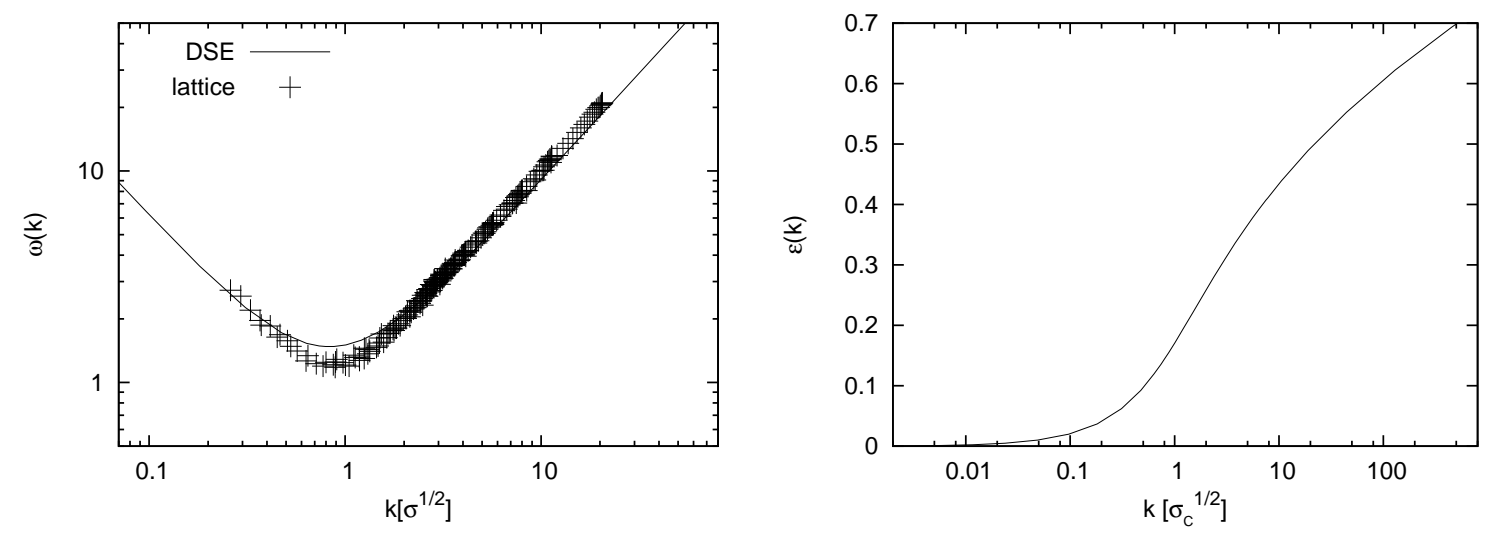

Figure 3: Left: Lattice data for $\omega(k)$, compared to the solution of the Dyson-Schwinger equations. Right: Dielectric function $\varepsilon(k)$.

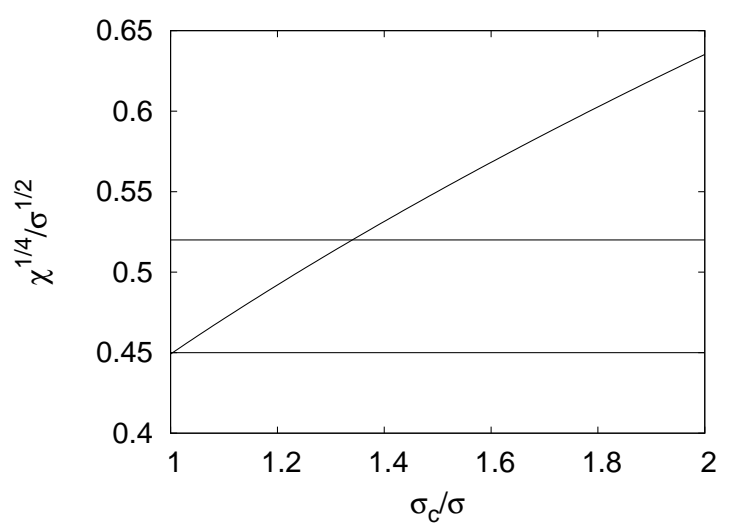

Figure 4: Topological susceptibility $\chi$ as a function of the ratio $\sigma_{c} / \sigma$.

with $M=0.88(1) \mathrm{GeV}$.

In ref. [11] it was shown that the inverse of the ghost form factor $d(k)$ can be identified as the dielectric function of the Yang-Mills vacuum

$$
\varepsilon(k)=d^{-1}(k)
$$

Fig. 3 (right panel) shows the so defined dielectric function. It satisfies $0<\varepsilon(k)<1$, which is a manifestation of anti-screening while in QED we have $\varepsilon(k)>1$, which corresponds to ordinary Debye screening. Furthermore, at zero momentum the dielectric function vanishes, showing that in the infrared the Yang-Mills vacuum behaves like a perfect colour dia-electric medium. The vanishing of the dielectric function in the infrared is not an artifact of our solutions of the DysonSchwinger equations but is guaranteed by the horizon condition, which is a necessary condition for the Gribov-Zwanziger confinement scenario. A perfect colour dia-electric medium $\varepsilon=0$ is nothing but a dual superconductor. (Here, "dual" refers to an interchange of electric and magnetic fields and charges.) Recall in an ordinary superconductor the magnetic permeability vanishes $\mu=0$. This shows that the Gribov-Zwanziger confinement scenario implies the dual Meissner effect [11]. 
In the Hamiltonian approach one finds the following expression for the topological susceptibility [14] (V: spatial volume)

$$
V \chi=\left(\frac{g^{2}}{8 \pi^{2}}\right)^{2}\left[\left\langle 0\left|\int B^{2}(x)\right| 0\right\rangle-2 \sum_{n} \frac{\left|\left\langle n\left|\int B \cdot \Pi\right| 0\right\rangle\right|^{2}}{E_{n}}\right] .
$$

Here $|n\rangle$ denotes the exact excited states of the Yang-Mills Hamiltonian with energies $E_{n}$. These eigenstates are of course not known. We work out the matrix elements in eq. (9) to two-loop order. In this order only two- and three-quasi gluon states contribute where the quasi gluons are defined as excitations of the vacuum $\langle 0 \mid A\rangle=\Psi(A)$ (1) with energy $\omega(k)$. The resulting expression for the topological susceptibility is ultraviolet divergent and needs renormalisation. For this aim we exploit the fact that $\chi$ vanishes to all order perturbation theory and renormalise the expression (9) for $\chi$ by subtracting each propagator by its perturbative expression. This renders $\chi$ (9) finite. Furthermore, since the momentum integrals in this expression are dominated by the infrared part we replace the coupling constant, which, in principle, should be the running one, by its infrared value. The results obtained in this way for the topological susceptibility are shown in Fig. 4 (right panel) as a function of the ratio $\sigma_{C} / \sigma$. Choosing $\sigma_{C}=1.5 \sigma$ which is the value favoured by the lattice calculation [7] we find for $S U(2)$ with $\sqrt{\sigma}=440 \mathrm{MeV}$

$$
\chi=(240 \mathrm{MeV})^{4} .
$$

This value is somewhat larger than the lattice prediction $\chi=(200-230 \mathrm{MeV})^{4}$.

The results obtained so far in the present approach are quite encouraging for further investigations. A natural next step would be the inclusion of dynamical quarks.

\section{References}

[1] D. Epple, H. Reinhardt, and W. Schleifenbaum, Phys. Rev. D75, 045011 (2007), hep-th/0612241.

[2] A. P. Szczepaniak and E. S. Swanson, Phys. Rev. D65, 025012 (2001), hep-ph/0107078.

[3] C. Feuchter and H. Reinhardt, Phys. Rev. D70, 105021 (2004), hep-th/0408236.

[4] W. Schleifenbaum, M. Leder, and H. Reinhardt, Phys. Rev. D73, 125019 (2006), hep-th/0605115.

[5] M. Leder, J. Pawlowski, H. Reinhardt and A. Weber, to be published

[6] C. Feuchter and H. Reinhardt, Phys. Rev. D77, 085023 (2008), 0711.2452.

[7] K. Langfeld and L. Moyaerts, Phys. Rev. D70, 074507 (2004), hep-lat/0406024.

[8] M. Quandt, G. Burgio, S. Chimchinda, and H. Reinhardt, PoS LAT2007, 325 (2007), arXiv:0710.0549 [hep-lat].

[9] R. Jackiw, I. Muzinich, and C. Rebbi, Phys. Rev. D17, 1576 (1978).

[10] G. Burgio, M. Quandt, and H. Reinhardt, (2008), 0807.3291.

[11] H. Reinhardt, Phys. Rev. Lett., 101, 061602 (2008) [arXiv:0803.0504 [hep-th]].

[12] S. L. Adler, Phys. Rev. 177, 2426 (1969).

[13] J. S. Bell and R. Jackiw, Nuovo Cim. A60, 47 (1969).

[14] D. R. Campagnari and H. Reinhardt, Phys. Rev. D 78, 085001 (2008) [arXiv:0807.1195 [hep-th]]

[15] D. Epple, H. Reinhardt, W. Schleifenbaum and A. P. Szczepaniak, Phys. Rev. D 77, 085007 (2008) [arXiv:0712.3694 [hep-th]]. 